\title{
Fatores de risco cardiovascular, saberes e práticas de cuidado de mulheres: possibilidade para rever hábitos ${ }^{a}$
}

\author{
Cardiovascular risk factors, knowledge and care practices of women: possibility to review habits \\ Factores de riesgo cardiovascular, conocimientos y prácticas de cuidado de las mujeres: posibilidad de \\ revisar hábitos
}

\author{
Gabriela Oliveira $^{1}$ (1) \\ Maria Denise Schimith ${ }^{1}$ (c) \\ Laís Mara Caetano da Silva ${ }^{1}$ (D) \\ Marta Regina Cezar-Vaz ${ }^{2}$ (b) \\ Fernanda Beheregaray Cabral ${ }^{3}$ (B) \\ Vanessa do Nascimento Silveira ${ }^{4}$ (1) \\ Luiza Camila Jerke ${ }^{5}$ (1]
}

1. Universidade Federal de Santa Maria,

Programa de Pós-Graduação em Enfermagem.

Santa Maria, RS, Brasil.

2. Universidade Federal do Rio Grande,

Programa de Pós-Graduação em Enfermagem. Rio Grande, RS, Brasil.

3. Universidade Federal de Santa Maria, Curso de Enfermagem. Palmeira das Missões, RS, Brasil.

4. Hospital São Francisco de Assis. Santa Maria, RS, Brasil.

5. Universidade Federal de Santa Maria, Curso de Enfermagem. Santa Maria, RS, Brasil.
Autor correspondente:

Gabriela Oliveira.

E-mail: gabrielabockenf@gmail.com

Recebido em 15/07/2021.

Aprovado em 22/10/2021.

DOI:https://doi.org/10.1590/2177-9465-EAN-2021-0281

\section{RESUMO}

Objetivo: investigar fatores de risco para doenças cardiovasculares e compreender as práticas de cuidado de mulheres Método: paralelo convergente misto. A etapa quantitativa foi transversal descritiva com formulário abordando características demográficas, socioeconômicas, fatores de risco cardiovascular, hábitos de vida e saúde, participaram 289 mulheres e aplicou-se estatística descritiva. A etapa qualitativa foi fundamentada na pesquisa participativa, exploratória descritiva, mediante entrevista semiestruturada, técnica de criatividade e sensibilidade e narrativas, com 30 mulheres. Resultados: identificaram-se fatores como sedentarismo $(60,9 \%)$, uso do anticoncepcional $(57,9 \%)$, estresse $(60,6 \%)$, depressão $(40,1 \%)$, obesidade $(38,8 \%)$, hipertensão arterial (33,6\%), consumo de bebida alcoólica (29,8\%), cigarro (16,6\%), dislipidemia (25,6\%) e diabetes (10,0\%) Quanto aos saberes e práticas de cuidado, as participantes mencionaram os fatores, porém algumas relataram realizar os cuidados, e outras não, mesmo reconhecendo os riscos. Assim, compartilharam a falta de tempo para realizar exercícios físicos, dificuldades para cuidar da alimentação e aderir ao tratamento anti-hipertensivo e, ainda, relataram sentirem-se estressadas Conclusão e implicações para a prática: identificaram-se fatores modificáveis e as mulheres possuíam conhecimento acerca destes, entretanto divergiam sobre os cuidados, demonstrando a importância de ações voltadas a essa população.

Palavras-chave: Doenças Cardiovasculares; Enfermagem; Estilo de Vida Saudável; Fatores de Risco; Mulheres.

\section{Abstract}

Objective: to investigate risk factors for cardiovascular disease and understand the care practices of women. Method: mixed convergent parallel. The quantitative stage was descriptive transversal with a form addressing demographic characteristics, socioeconomic, cardiovascular risk factors, lifestyle and health habits; 289 women participated and descriptive statistics were applied. The qualitative step was based on participatory research, exploratory descriptive, through semi-structured interviews, creativity and sensitivity technique and narratives, with 30 women. Results: factors were identified as sedentarism (60.9\%), use of contraceptives $(57.9 \%)$, stress $(60.6 \%)$, depression $(40.1 \%)$, obesity $(38.8 \%)$, high blood pressure $(33.6 \%)$, alcoho consumption (29.8\%), smoking (16.6\%), dyslipidemia (25.6\%), and diabetes (10.0\%). As for knowledge and care practices, the participants mentioned the factors, but some reported performing the care, and others did not, even though they recognized the risks. Thus, they shared the lack of time to perform physical exercises, difficulties to take care of the diet and adhere to antihypertensive treatment, and also reported feeling stressed. Conclusion and implications for practice: modifiable factors were identified and women had knowledge about these, however, they differed on the care, demonstrating the importance of actions aimed at this population.

Keywords: Cardiovascular Diseases; Nursing; Healthy Lifestyle; Risk Factors; Women.

\section{REsumen}

Objetivo: investigar los factores de riesgo para enfermedades cardiovasculares y comprender las prácticas de atención brindadas a las mujeres. Método: paralelo convergente mixto. La etapa cuantitativa fue transversal transcriptiva con formulario abordando características demográficas, socioeconómicas, factores de riesgo cardiovascular, hábitos de vida y salud. Participaron 289 mujeres y se aplicó estadística descriptiva. La etapa cualitativa fue fundamentada en la investigación participativa, exploratoria descriptiva, mediante entrevista semiestructurada, técnica de creatividad y sensibilidad y narrativas, con 30 mujeres. Resultados: se identificaron factores como sedentarismo (60,9\%), uso de anticonceptivos (57,9\%), estrés (60,6\%), depresión (40,1\%), obesidad $(38,8 \%)$, hipertensión arterial $(33,6 \%)$, consumo de bebidas alcohólicas $(29,8 \%)$, tabaquismo (16,6\%), dislipidemia $(25,6 \%)$ y diabetes $(10,0 \%)$. En cuanto a los conocimientos y prácticas de cuidado, las participantes mencionaron los factores, sin embargo, algunas reportaron realizar el cuidado y otras no, inclusive reconociendo los riesgos. Así, compartieron la falta de tiempo para la realización de actividades físicas, dificultades para cuidar su alimentación y la adherencia al tratamiento antihipertensivo e, inclusive, refirieron sentirse estresadas. Conclusión e implicaciones para la práctica: se identificaron factores modificables y las mujeres tenían conocimiento sobre ellos, sin embargo, divergieron sobre el cuidado, demostrando la importancia de las acciones dirigidas a esta población.

Palabras clave: Enfermedades Cardiovasculares; Enfermería; Estilo de Vida Saludable; Factores de Riesgo; Mujeres. 


\section{INTRODUÇÃO}

As doenças crônicas não transmissíveis (DCNTs) são consideradas um grave problema de saúde pública, responsáveis por 40 milhões de mortes, correspondendo a $70 \%$ do total de 56 milhões de óbitos no mundo, destacando-se, como principais, as doenças cardiovasculares (DCVs), responsáveis por $45 \%$ de todas as mortes por DCNTs ${ }^{1}$. Além de ser a principal causa de mortalidade no Brasil, as DCVs comprometem a qualidade de vida da população, cabendo destacar que a diminuição da mortalidade por DCVs acompanha melhores condições socioeconômicas em países de língua portuguesa como o Brasil, demonstrando o impacto das DCVs para além dos sistemas de saúde, abarcando a economia e seguridade social².

No entanto, algumas DCVs são, potencialmente, evitáveis e a Atenção Primária à Saúde (APS) constitui-se como importante cenário para prevenir e controlar, mediante ações de investigação e rastreamento dos fatores de risco (FRs) ${ }^{3}$. Ressalta-se que o desenvolvimento e o descontrole dos FRs para as DCVs aumentaram, consideravelmente, mudando até mesmo a prevalência das doenças, não figurando mais as transmissíveis, e sim as não transmissíveis ${ }^{4}$. Muitos FRs estão relacionados ao estilo de vida das pessoas. Logo, é indispensável identificá-los, bem como compreender os hábitos de vida, a fim de desenvolver ações de educação em saúde que permitam sensibilizar para a mudança comportamental. Instrumentalizar a população quanto aos fatores que levam às DCVs é primordial para que se reduzam os impactos destas 5 .

Revisões na literatura indicam a presença dos FRs cardiovascular na população feminina, entretanto, apontam para lacunas na realização de práticas de cuidado entre as mulheres, em especial frente as $\mathrm{DCVs}^{6,7}$. À vista disso, a investigação acerca dos fatores e comportamentos de risco é uma necessidade urgente, a fim de que sejam apresentados subsídios que contribuam com estratégias de prevenção e controle ${ }^{4}$.

O estudo em tela vai ao encontro dos Objetivos do Desenvolvimento Sustentável, relacionando-se ao item "Assegurar uma vida saudável e promover o bem-estar para todos, em todas as idades"8. Ademais, é referenciado na Agenda Nacional de Prioridades de Pesquisa em Saúde, no que se refere ao desenvolvimento de métodos de promoção à saúde, prevenção, diagnóstico, controle e tratamento precoce das DCNTs ${ }^{9}$.

Face ao exposto, desenvolveu-se o presente estudo com o intuito de responder às questões: quais os fatores de risco cardiovascular entre as mulheres atendidas na Atenção Primária à Saúde no interior do Rio Grande do Sul? E quais os saberes e práticas de cuidado dessas mulheres acerca dos fatores de risco para as doenças cardiovasculares? E o objetivo: investigar fatores de risco para doenças cardiovasculares e compreender as práticas de cuidado de mulheres.

\section{MÉTODO}

Os resultados divulgados no presente artigo são oriundos de uma Dissertação de Mestrado em Enfermagem. A pesquisa foi desenvolvida com o método misto, do tipo paralelo convergente (QUAN) + QUAL. Nessa representação, o parêntese significa que o método quantitativo está incorporado em um estudo maior, Projeto Matricial do Grupo de Pesquisa ao qual a Dissertação está vinculada, e o sinal de "+" representa que os dois métodos ocorreram simultaneamente. A combinação de diferentes métodos permite maior aprofundamento acerca do problema de estudo ${ }^{10}$. Dessa forma, a etapa quantitativa contemplou um estudo transversal descritivo e a qualitativa foi fundamentada na pesquisa participativa e desenvolvida de forma exploratória e descritiva.

O estudo foi realizado em unidades urbanas de APS em uma cidade no interior do estado do Rio Grande do Sul, no momento da coleta dos dados o município contava com 24 unidades urbanas e três unidades rurais de APS. As participantes foram mulheres atendidas em unidades urbanas da APS, que tivessem de 20 a 79 anos de idade, exceto as gestantes. A amostragem para a etapa quantitativa foi calculada, proporcionalmente, ao tamanho da população adscrita por região administrativa, de acordo com dados da Secretaria Municipal de Saúde, totalizando 289 participantes. As participantes foram captadas de forma aleatória e não sistemática nos serviços de saúde, enquanto aguardavam por atendimentos. Na etapa qualitativa, por sua vez, participaram 30 mulheres, selecionadas aleatoriamente, tendo sido encerrada a coleta no momento em que os objetivos da pesquisa foram alcançados. Não houve nenhuma recusa para participar da etapa de coleta de dados (entrevista semiestruturada conjugada à técnica de criatividade e sensibilidade), entretanto alguns contatos telefônicos para o convite não foram possíveis, pois as usuárias não atenderam a ligação, após diversas tentativas, ou pela inexistência do número. A coleta dos dados ocorreu de forma concomitante, de março de 2017 a maio de 2018. A captação das usuárias aconteceu de segunda a sexta-feira, no horário de funcionamento do serviço.

Para a etapa quantitativa, foi elaborado e entregue para cada coletador um manual, o qual possuía todas as informações acerca do estudo e dos procedimentos para coleta. Além disso, realizouse uma capacitação com duração de quatro horas para todos os coletadores e pesquisadores envolvidos, a fim de padronizar as aferições das variáveis do estudo. Ainda, foi pactuado que, para a investigação dos fatores de risco e medição dos dados antropométricos, cada coletador utilizaria seu material, como o esfigmomanômetro e estetoscópio (não foram identificados os fabricantes dos mesmos). Já as trenas utilizadas para medição da altura e circunferências foram disponibilizadas junto com o material da pesquisa e as balanças utilizadas eram as disponíveis em cada Unidade de Saúde. Foram convidadas todas as usuárias que estavam no serviço aguardando atendimento e a coleta dos dados se realizou por meio de um formulário. Este foi dividido em três módulos, a saber: 1: caracterização demográfica e informações sobre indicadores socioeconômicos (sexo, idade, escolaridade, ocupação/trabalho, estado civil, religião, etnia, deficiência física, número de pessoas que residem no domicílio, renda mensal familiar, condições do domicílio em que reside (próprio, cedido, 
alugado, outro - especifique), material do domicílio, esgotamento sanitário e abastecimento de água); 2: investigação dos fatores de risco e variáveis a serem avaliadas, como a pressão arterial e os dados antropométricos (peso, altura, índice da massa corpórea (IMC), circunferência abdominal, cintura e quadril), além do uso de anticoncepcional, uso do álcool e do cigarro, fumo passivo, uso de outras drogas, tratamento antidepressivo, estresse e realização de exercícios físicos; 3 : Escala de Risco de Framingham (ERF) para estratificar o risco cardiovascular (RCV) das usuárias, entretanto, o terceiro módulo não será descrito no presente artigo. Nesse sentido, para os valores obtidos a partir da investigação dos fatores de risco, utilizaram-se como referência para a classificação do IMC as Diretrizes Brasileiras de Obesidade ${ }^{11}$ e para a obesidade central, a estratificação de risco do Ministério da Saúde ${ }^{12}$. Já, para a quantidade de álcool ingerida, foi graduada conforme o limite máximo indicado pela Organização Mundial de Saúde ${ }^{13}$.

Ao tratar dos dados quantitativos, procedeu-se à dupla digitação independente no programa Epi Info 3.3.2 (Center for Disease Control and Prevention). Após, para realizar a conferência, os dados foram convertidos para uma planilha no software Microsoft Excele ajustaram-se as inconsistências existentes. Em seguida, procedeu-se à análise estatística descritiva, por meio do software $\mathrm{R}$, versão 3.4.3, com frequências absolutas e relativas para as variáveis categóricas e medidas de tendência central e dispersão para as variáveis contínuas. Ainda, analisou-se o Coeficiente de Variação de Pearson para verificar a distribuição dos dados, utilizando média e desvio padrão para as variáveis cujos dados tenham distribuição simétrica e mediana e intervalo interquartil para a variável com dados assimétricos.

$\mathrm{Na}$ etapa qualitativa, todas as coletas foram realizadas pela pesquisadora principal, participante do Grupo de Pesquisa ao qual o estudo é vinculado. As usuárias foram contatadas via telefone, oportunidade na qual agendou-se um encontro em local de preferência da participante, podendo ser a Unidade de Saúde ou o seu domicílio. Em situações em que a coleta ocorreu no domicílio, por vezes, a Agente Comunitária de Saúde permaneceu no local durante a produção dos dados. Destaca-se que se respeitou o consentimento das participantes e todos os aspectos éticos foram preservados.

A produção dos dados qualitativos ocorreu por meio de entrevista individual semiestruturada, conjugada à técnica de criatividade e sensibilidade (TCS) denominada Corpo Saber, realizada uma vez com cada participante. Para definir a ordem de utilização das técnicas foram realizados oito testes piloto. A partir dos testes piloto, definiu-se iniciar com a TCS, que é considerada uma produção artística na qual o corpo é a principal forma de acesso à experiência do cuidado ${ }^{14}$. Para a aplicação da técnica, disponibilizou-se um papel com a silhueta de uma mulher desenhada, que representava o corpo de cada uma, dessa forma, as participantes foram orientadas a desenhar/ escrever os cuidados que desenvolviam com o seu corpo. A questão geradora para a técnica foi "Quais os cuidados que você realiza com o seu corpo?". O objetivo era dimensionar as práticas de cuidado realizadas pelas mulheres, estabelecendo relações com o corpo. Ao finalizar, cada participante explicou, verbalmente, os cuidados descritos e/ou desenhados.

A seguir realizou-se a entrevista, oportunidade em que foram abordados o conhecimento das mulheres acerca dos FRs para as DCVs, a relação dos cuidados descritos na TCS com o desenvolvimento ou a prevenção das DCVs, as práticas de cuidado com a saúde, as potencialidades e barreiras para o desenvolvimento das práticas de cuidado. A produção dos dados qualitativos foi audiogravada e, após, transcrita na íntegra. Teve duração média de 30 minutos, e destaca-se que o tempo de gravação foi contabilizado a partir do momento em que a participante relatava a produção artística da TCS. Todo o tempo destinado para escrita/desenho não foi contabilizado e não se realizaram anotações durante a entrevista, uma vez que não eram objeto para a investigação.

A análise dos dados qualitativos foi realizada com base no referencial da pesquisa participativa, e se deu por meio da produção e devolução de narrativas. Para a elaboração das narrativas, realizaram-se diversas leituras, buscando aprofundar e compreender o material transcrito, identificando os núcleos argumentais que respondiam às questões de pesquisa e constituíram-se como fio condutor para a elaboração das narrativas. A produção da narrativa envolve a compreensão e interpretação, relacionando com o contexto social das participantes, ademais, buscou problematizar os hábitos e condutas, frente a vida ${ }^{15}$. Após a elaboração, as narrativas foram retornadas às participantes. Esse segundo encontro, no qual se deram o retorno e a leitura das narrativas às usuárias, também foi audiogravado e teve duração média de 40 minutos. Além de validar os dados, produziu efeitos de intervenção, permitindo à participante reconstituir e ressignificar a sua história e comportamentos, efeito esperado no uso desse método ${ }^{12}$. Por fim, por se tratar de um estudo de método misto, procedeu-se à interpretação e convergência dos resultados, relacionando e integrando os achados quantitativos e qualitativos.

O presente estudo respeitou os aspectos éticos da pesquisa com seres humanos, com apreciação e aprovação no Comitê de Ética em Pesquisa. Para tanto, houve a aprovação do Projeto Matricial em outubro de 2016, conforme o Parecer número 1.772.115, com o número do CAAE 60159416.2.0000.5346; e da Dissertação que integra o Projeto supracitado, em janeiro de 2018, Parecer número 2.464.295, CAAE 81455517.5.0000.5346. As participantes assinaram o Termo de Consentimento Livre e Esclarecido, que continha os objetivos da pesquisa e as razões para sua realização. $O$ anonimato das participantes foi preservado, por meio da utilização de códigos para a etapa quantitativa, e pela escolha de nomes de flores ou plantas para identificação na etapa qualitativa. Com o intuito de aprimorar a produção, utilizaram-se para a elaboração do presente artigo as diretrizes Revised Standards for Quality Improvement Reporting Excellence 2.0 (SQUIRE) ${ }^{16}$ e a Consolidated Criteria for Reporting Qualitative Research (COREQ) ${ }^{17}$. 


\section{RESULTADOS}

Das 289 mulheres, com média de idade de 44,0 anos (DP $15,35), 46,4 \%$ ( $n=134)$ estudaram por um período de 0 a 8 anos; $29,7 \%(n=86)$, de 9 a 11 anos; e 23,9\% ( $n=69)$, por 12 anos ou mais. Acrescenta-se que a média de anos de estudo foi de 9,5 anos (DP 4,2). Quanto à ocupação/trabalho, 57,5\% ( $n=165)$ possuíam renda própria, sendo aposentadas ou pensionistas, assalariadas ou autônomas, e as demais 42,5\% ( $n=122)$ eram desempregadas, do lar ou estudantes. Em dois formulários, a variável ocupação não foi preenchida de forma correta, variável que resultou em sua desconsideração.

Em relação ao estado civil, 53,0\% ( $n=153)$ possuíam companheiro, sendo casadas, legalmente, ou em união estável com mais de seis meses e união estável com menos de seis meses. Ainda, 47,0\% ( $n=136)$ não possuíam companheiro, sendo solteiras, separadas/desquitadas/divorciadas ou viúvas. Quanto à etnia, $64,7 \%(n=187)$ das mulheres se autodeclararam brancas; $20,5 \%(n=59)$, pardas; $13,8 \%(n=40)$, negras; e $1,0 \%(n=3)$, amarelas. No que concerne à renda (salário-mínimo regional no valor de $\mathrm{R} \$ 937,00)$, foi constatada a mediana de $\mathrm{R} \$ 1.874,00$ (IIQ 2.000,00). Com 31,5\% ( $n=91)$ recebendo mais de um até dois salários-mínimos; $20,8 \%(n=60)$, até um salário; $20,8 \%(n=60)$, mais de dois até três; $15,9 \%(n=46)$, mais de três até cinco; e, por fim, $11,0 \%(n=32)$ recebiam mais de cinco salários-mínimos vigentes no período da coleta de dados.

Considerando os FRs, o uso do anticoncepcional apresentou frequência de $57,9 \%(n=103)$ entre as mulheres em idade fértil (20 a 49 anos), conforme a tabela a seguir (Tabela 1).

Ao tratar do tipo de contraceptivo utilizado, mais de $70,0 \%$ $(n=77)$ das mulheres que faziam uso de contraceptivo optaram pelo oral, destas, $23,4 \%(n=18)$ faziam uso contínuo. Quanto ao anticoncepcional injetável, $25,2 \%(n=26)$ utilizavam e a maioria, $84,6 \%(n=22)$, optou pelo trimestral. Nos depoimentos, algumas participantes afirmaram já ter vivenciado ou ter conhecimento dos efeitos para a saúde relacionados ao uso do anticoncepcional.

[...]Ouvi dizer que, se a gente fica anos tomando esse remédio [anticoncepcional], acaba ficando com as veias trancadas, o sangue grosso, essas coisas[...] (PALMA50 anos, seis anos de estudo, assalariada, mais de um até dois salários-mínimos).

[...]Ela [médica] disse que, provavelmente, do remédio [anticoncepcional] que eu tomava que causou a trombose, aí, no momento que eu tive trombose, eles me proibiram, aí nunca mais tomei [anticoncepcional][...] (FLOR DO CAMPO - 52 anos, dois anos de estudo, assalariada, mais de um até dois salários-mínimos).

Outros fatores modificáveis, como o consumo de álcool e cigarro, foram identificados na pesquisa, conforme a Tabela 2.

No que diz respeito à ingestão de álcool e ao hábito de fumar, observou-se que a maior parte das mulheres não fazia uso de ambos. Entretanto, dentre as que ingeriam álcool, a faixa etária que se destaca é entre 20 a 29 anos. Entre as que fumavam, o maior número está entre as mulheres de 40 a 49 anos e, em seguida, as mulheres com idade entre 20 a 29 anos. Ademais, as participantes demonstraram reconhecer o consumo de álcool e cigarro, quando questionadas a respeito dos fatores de risco para DCVs.

[...]A bebida de álcool, o cigarro [considera como fatores de risco], coisa que estou recém, parando de fumar, mas estou tentando[...] (COMIGO NINGUÉM PODE - 61 anos, cinco anos de estudo, autônoma, até um salário-mínimo).

Em relação aos distúrbios psicológicos, mais de 40,0\% $(n=116)$ das participantes afirmaram realizar ou já terem realizado tratamento para depressão. Além disso, mais de $60,0 \%(n=175)$ das mulheres sentiam-se estressadas no momento da coleta de dados. Alguns depoimentos reiteram os achados da etapa quantitativa.

[...]Eu sou bastante ansiosa, estressada, depressão e essas coisas todas acabam afetando todo o corpo, na minha opinião[...] (MARACUJÁ - 40 anos, 12 anos de estudo, assalariada, mais de três até cinco salários-mínimos).

Tabela 1. Distribuição das participantes segundo o uso de anticoncepcional e faixa etária. Rio Grande do Sul, Brasil, 2018.

\begin{tabular}{|c|c|c|c|c|}
\hline \multirow{2}{*}{ Faixa etária (anos) } & \multicolumn{3}{|c|}{ Anticoncepcional } & \multirow{2}{*}{ Total } \\
\hline & Hormonal & Outro & Não faz uso & \\
\hline $20-29$ & 40 & 1 & 22 & 63 \\
\hline $30-39$ & 36 & 2 & 16 & 54 \\
\hline $40-49$ & 22 & 2 & 37 & 61 \\
\hline $50-59$ & 5 & 0 & 57 & 62 \\
\hline $70-79$ & 0 & 0 & 19 & 19 \\
\hline Total & 103 & 5 & 181 & 289 \\
\hline
\end{tabular}

Fonte: resultados da pesquisa. 
[...]Do sistema nervoso que abala muito, a pessoa fica muito preocupada, agitada, estressada e aí as consequências podem ser até um AVC[...] (LíRIO - 72 anos, um ano de estudo, pensionista, mais de dois até três salários-mínimos).

O excesso de peso e/ou a obesidade constituem de forma isolada um risco à saúde, e um dos principais FRs cardiovascular. Conforme o cálculo do IMC, observa-se que a maioria das participantes foi classificada como obesa, $38,8 \%(n=112)$ (grau I, II e III), variável apresentada a seguir, na Tabela 3.

Entre as mulheres classificadas com obesidade ( $n=112)$, 0 maior número estava com obesidade grau $\mathrm{I}$, que corresponde a $56,2 \%(n=63), 43,8 \%(n=49)$ estavam com obesidade grau II ou grau III. Procedendo-se à análise a partir da faixa etária, percebeu-se que a quantidade de mulheres magras ou eutróficas tende a diminuir conforme $o$ avanço da idade, visto que mulheres com 20 a 29 anos de idade, quando comparadas às mulheres de outras faixas etárias, apresentaram maiores frequências nas categorias "magra ou baixo peso" e "normal ou eutrófico". A média do IMC foi de 29,07 kg (DP 6,3). Ao tratar dos depoimentos, as mulheres reconheciam a alimentação inadequada como FR para as DCVs, entretanto a maioria relatava dificuldades para realizar os cuidados necessários.

[...] A alimentação, o fato de eu comer muita besteira, acho que prejudica bastante, seria um fator. Ee por preguiça, preguiça de comer bem, de comer saudável, eu só quero chegar no mercado e, olha aí, ó, um salgadinho, vou ali e pego aquele salgadinho que é mais fácil de comer, ou antes de vir para universidade também [...] (AZALEIA 22 anos, 15 anos de estudo, estudante, mais de um até dois salários-mínimos).

[...] A minha alimentação é zero, pouco líquido, muito refrigerante, a própria fritura, a má alimentação, que eu gosto de carne gorda, gosto de fritura, que é uma das coisas que poderia contribuir [para o desenvolvimento das

Tabela 2. Distribuição das participantes segundo o uso de álcool e cigarro por faixa etária. Rio Grande do Sul, Brasil, 2018.

\begin{tabular}{|c|c|c|c|c|c|}
\hline \multirow[b]{2}{*}{ Faixa etária (anos) } & \multicolumn{3}{|c|}{ Álcool } & \multicolumn{2}{|c|}{ Cigarro } \\
\hline & Não faz uso & $\begin{array}{l}\text { Menos de } 30 \\
\text { gramas por dia }\end{array}$ & $\begin{array}{c}\text { Mais de } 30 \text { gramas } \\
\text { por dia }\end{array}$ & Não faz uso & Faz uso \\
\hline $20-29$ & 29 & 31 & 3 & 52 & 11 \\
\hline $30-39$ & 33 & 19 & 2 & 47 & 7 \\
\hline $40-49$ & 44 & 16 & 1 & 47 & 14 \\
\hline $50-59$ & 52 & 10 & 0 & 53 & 9 \\
\hline $60-69$ & 28 & 2 & 0 & 26 & 4 \\
\hline 70-79 & 17 & 2 & 0 & 16 & 3 \\
\hline Total & 203 & 80 & 6 & 241 & 48 \\
\hline
\end{tabular}

Fonte: resultados da pesquisa.

Tabela 3. Distribuição das participantes segundo a classificação do índice de massa corporal e faixa etária. Rio Grande do Sul, Brasil, 2018.

\begin{tabular}{|c|c|c|c|c|c|c|c|}
\hline \multirow[b]{2}{*}{ Faixa Etária } & \multicolumn{6}{|c|}{ Índice de Massa Corporal } & \multirow[b]{2}{*}{ Total } \\
\hline & $\begin{array}{c}\text { Magra ou } \\
\text { Baixo Peso } \\
(<18,5)\end{array}$ & $\begin{array}{c}\text { Normal ou } \\
\text { Eutrófico } \\
(18,6-24,9)\end{array}$ & $\begin{array}{l}\text { Sobrepeso } \\
(25,0-29,9)\end{array}$ & $\begin{array}{c}\text { Obesidade } \\
\text { Grau I }(30,0- \\
34,9)\end{array}$ & $\begin{array}{c}\text { Obesidade } \\
\text { Grau II } \\
\text { (severa) } \\
(35,0-39,9)\end{array}$ & $\begin{array}{c}\text { Obesidade } \\
\text { Grau III } \\
\text { (mórbida) } \\
(>40,0)\end{array}$ & \\
\hline $20-29$ & 2 & 27 & 14 & 11 & 3 & 6 & 63 \\
\hline $30-39$ & 1 & 15 & 15 & 14 & 7 & 2 & 54 \\
\hline $40-49$ & 0 & 15 & 18 & 15 & 8 & 5 & 61 \\
\hline $50-59$ & 1 & 14 & 23 & 14 & 6 & 4 & 62 \\
\hline $60-69$ & 1 & 7 & 7 & 7 & 4 & 4 & 30 \\
\hline $70-79$ & 0 & 4 & 13 & 2 & 0 & 0 & 19 \\
\hline Total & 4 & 83 & 90 & 63 & 28 & 21 & 289 \\
\hline
\end{tabular}

Fonte: resultados da pesquisa. 
DCVs][...] (PRIMAVERA - 45 anos, 13 anos de estudo, estudante, mais de três até cinco salários-mínimos).

Percebeu-se que, além da alimentação inadequada, o sedentarismo também foi citado como um FR. Ressalta-se que mais de $60,0 \%$ ( $n=176$ ) das mulheres estavam sedentárias no momento da coleta de dados. Nos depoimentos, algumas mulheres mencionaram a falta de tempo como um fator impeditivo para a realização de exercícios físicos.

[...]Até eu tinha que fazer [exercício físico - caminhadas], mas não faço porque eu não tenho tempo, meu marido trabalha para fora e eu estou cuidando o bar, e o marido da minha filha se empregou também, então, ficou só nós duas no bar e na lavagem, então, eu não consigo fazer[...] (ORQUÍDEA - 45 anos, quatro anos de estudo, autônoma, mais de dois até três salários-mínimos).

[...]Eu estava fazendo academia, mas eu parei, dei até uma "engordadinha", parei devido ao novo emprego, porque os horários estavam ficando meio complicados de conseguir combinar, e de manhã eu fico com o meu filho. Eu parei por falta de tempo[...] (RÁFIA - 24 anos, 12 anos de estudo, assalariada, mais de três até cinco salários-mínimos).

A média constatada para a Pressão Arterial Sistólica (PAS) foi de 124,7 mmHg (DP 22,7) e para a Pressão Arterial Diastólica (PAD), $80,0 \mathrm{mmHg}$ (DP 13,6). Entretanto, 33,6\% ( $n=97)$ das participantes referiram possuir o diagnóstico médico de Hipertensão Arterial (HA) e, nos depoimentos, mesmo reconhecendo como um FR para as DCVs, algumas demonstraram pouca adesão ao tratamento.

[...]Ele [médico] disse que minha pressão é alta, aquele dia que eu fui consultar minha pressão estava 17 por 10 [170/100 mmHg] e ele disse que estava superalta e eu tinha parado de tomar a medicação por conta minha[...] (DAMA DA NOITE - 45 anos, três anos de estudo, desempregada, mais de um até dois salários-mínimos).

[...]Da pressão eu tomava [medicação], quando eu estava bem, eu não tomava, só quando eu estava meio zonza eu tomava, mas agora eu estou assustada[...] (DINHEIRINHO - 63 anos, nove anos de estudo, aposentada, mais de um até dois salários-mínimos).

Outros FRs foram evidenciados na população estudada, como dislipidemia em $25,6 \%(n=74)$ das participantes e o diabetes mellitus (DM) em 10,0\% $(n=29)$ das mulheres. $\mathrm{Na}$ etapa qualitativa, algumas participantes mencionaram práticas de cuidado considerando estes fatores.

[...]Como bastante verdura, verdura aqui em casa não falta, fritura também não faço, porque, quando fiz uma avaliação para fazer uma cirurgia, fiz uns exames e eu não tinha colesterol, mas aí apareceu, então, fritura não faço[...] (FLOR DE MAIO - 65 anos, seis anos de estudo, aposentada, mais de um até dois salários-mínimos).

[...] Na alimentação, eu me cuido, procuro não comer muita coisa gordurosa, muitas massas, porque eu sou diabética, então, procuro me cuidar [...] fui até a nutricionista [...] E os cuidados com os pés, porque eu tenho os problemas com os pés, como sou diabética[...] (FLOR DO CAMPO -52 anos, dois anos de estudo, assalariada, mais de um até dois salários-mínimos).

Evidenciou-se a presença de diversos FRs modificáveis na população estudada. Além disso, foi possível perceber que muitos hábitos desenvolvidos pelas mulheres favorecem o desenvolvimento e agravamento desses fatores e, consequentemente, impactam em complicações decorrentes das DCVs.

\section{DISCUSSÃO}

Identificou-se que quanto maior a idade das mulheres, menor a escolaridade, pois $76,0 \%(n=102)$ das mulheres com mais de 40 anos estudaram de 0 a 8 anos, ou seja, completaram no máximo o ensino fundamental. Tal situação demonstra uma condição histórica, visto que em décadas passadas a educação atribuída às mulheres raramente envolvia conhecimentos básicos, como a alfabetização, limitava-se aos "bons costumes domésticos"18.

Contudo, no que diz respeito à ocupação/trabalho, evidenciouse a inserção da mulher no mercado de trabalho, já que a maioria das participantes possuía renda própria, oriunda de aposentadoria/ pensão, ou ainda do trabalho autônomo ou assalariado. Percebese a inserção feminina no âmbito da economia e da sociedade em geral, não sendo mais a atividade do lar sua única ocupação. A inserção da mulher no mercado de trabalho apresentou um aumento nas últimas décadas, o que torna necessário atentar-se ao fato de que as diversas atividades podem gerar sobrecarga na rotina feminina, pois, na maioria das vezes, continua sendo a principal provedora dos cuidados familiares e executora das tarefas relacionadas ao domicílio ${ }^{19}$.

A renda, em salários-mínimos, demonstra que a maioria das participantes estava recebendo de um a dois salários, seguindose até um salário-mínimo, e mais de dois a três salários-mínimos. Nesse sentido, o Instituto Brasileiro de Geografia e Estatística traz que as mulheres possuem maior nível de escolaridade, trabalham mais tempo e ganham, em média, $76,5 \%$ do rendimento dos homens ${ }^{20}$. As mulheres possuem, muitas vezes, jornada dupla ou tripla, e renda menor, quando comparadas aos homens, o que evidencia a necessidade de refletir e buscar formas para mudar essa realidade, construída historicamente, por meio de ações de incentivo ao empoderamento feminino, em busca de sua valorização e da efetivação de seus direitos.

Ao tratar dos FRs, evidenciaram-se os fatores modificáveis, influenciados pelas condições e hábitos de vida, condicionados pelas práticas de cuidado. Infere-se, com isso, ser primordial 
que as pessoas desenvolvam um estilo de vida saudável ou, ainda, quando necessário, busquem realizar mudanças no comportamento diário, com intuito de controlar e prevenir o surgimento e progressão das $\mathrm{DCVs}^{7}$.

Mulheres em idade reprodutiva, que fazem uso de contraceptivo, constituíram 57,9\% ( $n=103)$ da amostra. Embora os contraceptivos sejam uma estratégia para o planejamento familiar, uma opção para o controle da fertilidade, estudo de revisão evidenciou a associação entre alterações pressóricas e uso de anticoncepcionais hormonais combinados. Nesse sentido, o estudo destaca que, independente da concentração, a presença de estrogênio exógeno na circulação sanguínea ativa o sistema renina-angiotensina-aldosterona, ocasionando retenção de água e sódio ${ }^{21}$. Acrescem-se as evidências de outro estudo que investigou o perfil de risco cardiovascular de mulheres jovens e constatou que a maioria das mulheres de $20 \mathrm{a}$ 50 anos apresentava um ou mais FRs para as DCVs, reforçando a importância de identificar os FRs e buscar a prevenção das DCVs com a população feminina ainda na fase reprodutiva ${ }^{22}$.

Foi identificado que algumas participantes possuíam conhecimentos e experiências acerca das possíveis complicações decorrentes do uso do contraceptivo, principalmente, relacionadas ao sistema circulatório. Dessa forma, evidencia-se a importância de considerar o histórico e os FRs no momento da indicação de uso ou não do anticoncepcional, diminuindo a influência sobre o sistema cardiovascular ${ }^{21}$. Ressalta-se que, além de orientar a respeito da utilização e, principalmente, dos possíveis efeitos adversos decorrentes de seu uso, que os prescritores cuidem singularmente das mulheres, visando a benefícios efetivos e à redução de danos ${ }^{22}$.

Dentre os FRs modificáveis para DCVs, tem-se também o alcoolismo e o tabagismo. A maioria das participantes não fazia uso de álcool e tabaco, entretanto, dentre as que possuíam esses hábitos, as mulheres com idade entre 20 a 29 anos se destacaram, em especial por apresentarem a maior frequência para o uso do álcool e a segunda maior frequência para o hábito de fumar. Corroborando com esse achado, dados internacionais identificaram aumento no consumo de álcool entre mulheres jovens ${ }^{23}$. Nesse sentido, ressalta-se a importância de expandir as ações de promoção à saúde para outros contextos, além dos serviços de saúde, incluindo escolas, universidades e eventos que tenham maior concentração de jovens.

O transtorno depressivo constitui-se como um importante problema de saúde pública, devido à elevada prevalência, à repercussão na saúde e no bem-estar, além do impacto psicossocial. Evidências científicas apontam que o transtorno depressivo pode influenciar no desenvolvimento e agravamento de quadros clínicos como diabetes, cardiopatias, obesidade e disfunções oncológicas ${ }^{24}$. Destaca-se também o estresse, fortemente, evidenciado nos resultados deste estudo e, predominantemente, declarado entre mulheres com faixa etária socialmente e economicamente ativa.

Esse achado reforça, novamente, a mudança do papel feminino na sociedade e na família, visto que as mulheres passaram a ser solicitadas a contribuir financeiramente no sustento da família, permanecendo com as responsabilidades da maternidade, do lar e as cobranças sociais. Essa sobrecarga de demandas e trabalho pode resultar em níveis de estresse elevado $\mathrm{e}^{19}$, consequentemente, expõe as mulheres a diversos riscos para a saúde e para o bem-estar. Nos depoimentos, as mulheres reconheceram o transtorno depressivo e o estresse como FRs para DCVs. Reitera-se que a sobrecarga de demandas também foi constatada, sendo um dos motivos para que as mulheres apresentassem dificuldades de realizar as práticas de cuidado com a saúde.

Os principais riscos modificáveis para a saúde da população são a obesidade e o sedentarismo. Vivencia-se uma pandemia de excesso de peso, onde as ações em saúde se encontram em uma perspectiva de tratamento e as voltadas à prevenção primária, com tímida implementação ${ }^{25}$. Houve um aumento de obesidade de $6,4 \%$ para $14,9 \%$ entre as mulheres, no período de 1975 a 2014, com tendência a ser mantida em 2025, quando a prevalência será maior que $21 \%$ entre as mulheres no cenário mundial26.

Esse preocupante panorama associa-se aos hábitos alimentares da sociedade moderna e à inatividade física. A maioria das participantes reconheceu os fatores elencados como prejudiciais à saúde, porém relataram dificuldades para realizar os cuidados necessários. Essas dificuldades envolvem, principalmente, a redução na ingestão de alimentos ricos em gorduras, o excesso de sal, carboidratos e alimentos industrializados, além da falta de exercícios físicos. Estudo que avaliou o impacto dos alimentos ultraprocessados no perfil nutricional da dieta no Brasil evidenciou a influência desfavorável desses alimentos na qualidade da alimentação, devido à elevação energética e dos teores de gorduras saturadas, gorduras trans e açúcares associados, bem como a diminuição dos teores de potássio e fibras ${ }^{27}$. Com isso, verifica-se a urgência em repensar as ações voltadas à prevenção da obesidade e do sedentarismo, que podem ser desenvolvidas a partir de orientações e elaboração de plano de cuidados de forma conjunta, mas também com políticas públicas voltadas à indústria alimentícia.

A HA apresentou maior frequência entre mulheres com mais de 50 anos, o que coaduna com as diretrizes brasileiras. A doença está associada ao aumento da idade devido, principalmente, ao envelhecimento vascular, em associação a outros fatores como a genética, hábitos alimentares desequilibrados, consumo de álcool, cigarro e sedentarismo ${ }^{28}$. As participantes identificaram a HA como um FR para as DCV s e algumas relataram apresentar essa condição. Quanto aos cuidados, relataram ter dificuldades para o controle dos níveis pressóricos e realização do tratamento contínuo, interrompendo o uso do fármaco.

Nesse sentido, a não adesão ao tratamento anti-hipertensivo tem-se apresentado como um grande problema de saúde pública, realidade reforçada por uma revisão sistemática que evidenciou que mais de $45 \%$ das pessoas estudadas não realizavam o tratamento medicamentoso corretamente ${ }^{29}$. A HA é uma condição crônica e necessita, além do tratamento medicamentoso, cuidados 
diários para o controle. Nesse contexto, uma das atribuições da equipe de APS é o acompanhamento da saúde dos usuários, o que oportuniza a identificação de possíveis dificuldades com os cuidados e possibilita a realização de orientações efetivas.

A dislipidemia também apresentou maior frequência entre as mulheres com mais de 50 anos. Nessa faixa etária, as mulheres encontram-se no climatério, período em que ocorrem alterações metabólicas, como a dislipidemia ${ }^{30}$. Soma-se, ainda, o DM que, além de ser um FR controlável para as DCVs, também é uma condição crônica que, quando não tratada, compromete a saúde das mulheres e, consequentemente, impacta na qualidade de vida. Os resultados apontaram a prevalência do DM autorreferido entre $10,0 \%$ das participantes. Percebe-se a relevância desse dado, uma vez que a prevalência de DM para mulheres de 20 a 79 anos foi estimada em $8,4 \%$ e prevê-se que aumente para 9,7\%, sendo a realidade identificada no estudo superior ${ }^{31}$.

Frente ao exposto, destaca-se a importância de desenvolver ações que promovam a prevenção e o controle dos FRs para as DCVs, além de atividades educativas que busquem sensibilizar e contribuam com o empoderamento das usuárias, oportunizando a implantação de diferentes estratégias de cuidado, como dieta saudável, realização de exercícios físicos e políticas públicas, essenciais para a qualidade de vida. Acrescenta-se a importância de realizar ações que controlem os FRs e foquem, sobretudo, na prevenção, uma vez que se apresentam em crescimento na população, se constituindo como a principal causa de óbitos, afastamento das atividades, além de prejuízo econômico, social e afetivo ${ }^{25}$.

\section{CONCLUSÃO E IMPLICAÇÕES PARA A PRÁTICA}

Identificou-se a presença de FRs modificáveis entre as mulheres, tais como sedentarismo, uso do anticoncepcional, estresse, depressão, obesidade, HA, consumo de bebida alcoólica, cigarro, dislipidemia e DM. Além disso, compreendeuse que algumas participantes realizavam práticas de cuidado relacionadas ao controle dos FRs, como interromper o uso de anticoncepcional, cessar o tabagismo e ter hábitos alimentares saudáveis, entretanto, outras participantes desenvolviam hábitos que contribuíam para o desenvolvimento e agravamento das DCVs, por exemplo, estresse, não realização de exercícios físicos em virtude de falta de tempo, dificuldades para realizar os cuidados com a alimentação e dificuldades na adesão ao tratamento para HA. Essa compreensão só foi possível devido ao delineamento de método misto, que proporcionou melhor entendimento do fenômeno estudado. Ademais, a influência dos hábitos de vida e dos cuidados em relação ao manejo dos FRs reforçou a necessidade de planejar ações em saúde, direcionadas à prevenção e controle das DCVs, considerando, especialmente, o contexto atual da mulher na sociedade. Por fim, acrescenta-se que a análise qualitativa mediante elaboração e devolução de narrativas possibilitou o repensar das participantes sobre seus hábitos e cuidados, como um processo de sensibilização, se mostrando como um método com potencial para contribuir com o aprimoramento da pesquisa em saúde e enfermagem.
A limitação do estudo se relaciona, especialmente, à dificuldade em obter os resultados dos exames laboratoriais, o que impossibilitou a estratificação do risco cardiovascular e, consequentemente, a identificação de alguns desses fatores. Acredita-se que, ao identificar os FRs para as DCVs presentes entre as mulheres, compreender os hábitos promotores da saúde e os desafios presentes na efetivação das práticas de cuidado, é possível subsidiar a prática de enfermagem em ações de educação, promoção em saúde e prevenção de agravos. A enfermagem pode auxiliar as mulheres no planejamento do cuidado para controlar os FRs, discutindo com elas a anticoncepção, o tempo para exercícios, as adaptações da alimentação, buscando, principalmente, o cuidado longitudinal e singular. Além disso, os achados do presente estudo podem contribuir com a realização de estudos futuros que venham a intervir e promover o cuidado direcionado às mulheres com FRs cardiovascular.

\section{FINANCIAMENTO}

Apoio financeiro da Coordenação de Aperfeiçoamento de Pessoal de Nível Superior - Brasil (CAPES) - Código de Financiamento 001. Bolsa de Mestrado concedida a Gabriela Oliveira, sob orientação da Profa. Dra. Maria Denise Schimith.

\section{CONTRIBUIÇÕES DOS AUTORES}

Desenho do estudo. Gabriela Oliveira. Maria Denise Schimith. Laís Mara Caetano da Silva. Vanessa do Nascimento Silveira.

Coleta de dados. Gabriela Oliveira. Maria Denise Schimith. Laís Mara Caetano da Silva. Vanessa do Nascimento Silveira.

Análise de dados. Gabriela Oliveira. Maria Denise Schimith. Laís Mara Caetano da Silva. Marta Regina Cezar-Vaz. Fernanda Beheregaray Cabral. Luiza Camila Jerke

Interpretação dos resultados. Gabriela Oliveira. Maria Denise Schimith. Laís Mara Caetano da Silva. Vanessa do Nascimento Silveira. Marta Regina Cezar-Vaz. Fernanda Beheregaray Cabral. Luiza Camila Jerke

Redação e revisão crítica do manuscrito. Gabriela Oliveira. Maria Denise Schimith. Laís Mara Caetano da Silva. Marta Regina Cezar-Vaz. Fernanda Beheregaray Cabral. Vanessa do Nascimento Silveira. Luiza Camila Jerke

Aprovação da versão final do artigo. Gabriela Oliveira. Maria Denise Schimith. Laís Mara Caetano da Silva. Marta Regina Cezar-Vaz. Fernanda Beheregaray Cabral. Vanessa do Nascimento Silveira. Luiza Camila Jerke

Responsabilidade por todos os aspectos do conteúdo e a integridade do artigo publicado. Gabriela Oliveira. Maria Denise Schimith. Laís Mara Caetano da Silva. Marta Regina Cezar-Vaz. Fernanda Beheregaray Cabral. Vanessa do Nascimento Silveira. Luiza Camila Jerke

\section{EDITOR ASSOCIADO}

Stela Maris de Melo Padoin (1) 


\section{EDITOR CIENTÍFICO}

\author{
Ivone Evangelista Cabral (1)
}

\section{REFERÊNCIAS}

1. WHO:World Health Organization. World health statistics 2017: monitoring health for the SDGs, Sustainable Development Goals [Internet]. Geneva: WHO;2017 [citado 10 fev 2019]. Disponível em: http://apps. who.int/iris/ bitstream/10665/255336/1/9789241565486-eng.pdf

2. Nascimento BR, Brant LCC, Oliveira GMM, Malachias MVB, Reis GMA, Teixeira RA et al. Cardiovascular disease epidemiology in portuguesespeaking countries: data from the Global Burden of Disease, 1990 to 2016. Arq Bras Cardiol. 2018;110(6):500-11. http://dx.doi.org/10.5935/ abc.20180098. PMid:30226906.

3. Lentsck MH, Mathias TAF. Hospitalizations for cardiovascular diseases and the coverage by the family health strategy. Rev Lat Am Enfermagem. 2015;23(4):611-9. http://dx.doi.org/10.1590/0104-1169.0078.2595 PMid:26444162.

4. Ferreira SRG, Chiavegatto ADP Fo, Lebrão ML, Duarte YAOD, Laurenti R Cardiometabolic diseases. Rev Bras Epidemiol.2018;21(Supl.2):e180008. http://dx.doi.org/10.1590/1980-549720180008.supl.2. PMid:30726353.

5. Bonotto GM, Mendoza-Sassi RA, Susin LRO. Knowledge of modifiable risk factors for cardiovascular disease among women and the associated factors: a population-based study. Cien Saude Colet. 2016;21(1):293-302. http://dx.doi.org/10.1590/1413-81232015211.07232015. PMid:26816186.

6. Oliveira G, Schimith MD, Silveira VN. Fatores de risco cardiovascular em mulheres: revisão integrativa da literatura. Enfermagem Brasil. 2019;18(6):799-815. http://dx.doi.org/10.33233/eb.v18i6.2140.

7. Oliveira G, Schimith MD, Ressel LB, Prates LA, Munhoz OL, Champe TS. Women with cardiovascular risk: review of research from brazilian graduate programs. Rev Bras Promoç Saúde. 2018;31(2):1-11. http:// dx.doi.org/10.5020/18061230.2018.6938.

8. $\quad$ Organização das Nações Unidas, Centro de Informação das Nações Unidas para o Brasil. Transformando nosso mundo: a agenda 2030 para o desenvolvimento sustentável [Internet]. Brasília: Centro de Informação das Nações Unidas para o Brasil; 2015.41 p. [citado 12 fev 2019]. Disponível em: https://sustainabledevelopment.un.org/content/documents/21252030\%20 Agenda\%20for\%20Sustainable\%20Development\%20web.pdf

9. Ministério da Saúde (BR), Secretaria de Ciência, Tecnologia e Insumos Estratégicos, Departamento de Ciência e Tecnologia. Agenda nacional de prioridades de pesquisa em saúde [Internet]. $2^{\mathrm{a}}$ ed. Brasília: Ministério da Saúde;2015.70 p. [citado 12 fev 2019]. Disponível em: http://bvsms.saude. gov.br/bvs/publicacoes/agenda_nacional_prioridades_2ed_4imp.pdf

10. Creswell JW, Clark VLP. Pesquisa de métodos mistos. $2^{\underline{a}}$ ed. Porto Alegre: Penso; 2013. $288 \mathrm{p}$.

11. ABESO: Associação Brasileira para o Estudo da Obesidade e da Síndrome Metabólica. Diretrizes brasileiras de obesidade 2016 [Internet]. $4^{a}$ ed. São Paulo: ABESO; 2016 [citado 12 fev 2019]. Disponível em: www.abeso. org.br/uploads/downloads/92/57fccc403e5da.pdf

12. Ministério da Saúde (BR), Secretaria de Atenção à Saúde, Departamento de Atenção Básica. Prevenção clínica de doenças cardiovasculares, cerebrovasculares e renais [Internet]. Brasília: Ministério da Saúde;2006. 56 p. [citado 12 fev 2019]. Disponível em: http://bvsms.saude.gov.br/bvs/ publicacoes/abcad14.pdf

13. WHO:World Health Organization. Global status report on alcohol and health 2014 [Internet]. Geneva:WHO;2014 [citado 12 fev 2019]. Disponível em: https://apps.who.int/iris/bitstream/handle/10665/112736/9789240692763_ eng.pdf?sequence $=1$

14. Cabral IE, Neves ET. Pesquisar com o método criativo e sensível na enfermagem: fundamentos teóricos e aplicabilidade. In: Lacerda MR, Costenaro RGS, editores. Metodologias da pesquisa para a enfermagem e saúde. 1를. Porto Alegre: Moriá; 2016. Cap. 12, p. 325-50.

15. Onocko-Campos RT. Fale com eles! O trabalho interpretativo e a produção de consenso na pesquisa qualitativa em saúde: inovações a partir de desenhos participativos. Physis Rev Saúde Colet [Internet]. 2011; [citado
12 fev 2019];21(2):1269-86. Disponível em: http://www.scielo.br/pdf/ physis/v21n4/a05v21n4.pdf

16. Ogrinc G, Davies L, Goodman D, Batalden P, Davidoff F, Stevens D. SQUIRE 2.0 [Standards for QUality Improvement Reporting Excellence] revised publication guidelines from a detailed consensus process. Perm J. 2015;19(4):65-70. http://dx.doi.org/10.7812/TOP/15-141.PMid:26517437.

17. Tong A, Sainsbury P, Craig J. Consolidated criteria for reporting qualitative research (COREQ): a 32-item checklist for interviews and focus groups. Int J Qual Health Care. 2007;19(6):349-57. http://dx.doi.org/10.1093/ intqhc/mzm042. PMid:17872937.

18. Queiroz JV, Gonçalvez LA, Kruger GN. Análise do grau de escolaridade das mulheres no Brasil. Anais Eletrônicos do Seminário Internacional Fazendo Gênero 11 \& 13th Women's Worlds Congress [Internet]; 2017 Florianópolis (SC), Brasil. Florianópolis: UFSC; 2017 [citado 18 fev 2019] Disponível em: http://www.wwc2017.eventos.dype.com.br/resources/ anais/1499435808_ARQUIVO_Artigo\%20(Recuperado).pdf

19. Costa FA. Mulher, Trabalho e Família: os impactos do trabalho na subjetividade da mulher e em suas relações familiares. Rev Grad Psicol [Internet]. 2018; [citado 18 fev 2019];3(6):434-52. Disponível em: http:// periodicos.pucminas.br/index.php/pretextos/article/view/15986

20. IBGE: Instituto Brasileiro de Geografia e Estatística. Estatísticas de gênero: indicadores sociais das mulheres no Brasil [Internet]. 2018 [citado $23 \mathrm{fev}$ 2019]. Disponível em: https://biblioteca.ibge.gov.br/visualizacao/livros/ liv101551_informativo.pdf

21. Ribeiro CCM, Shimo AKK, Lopes MHBM, Lamas JLT. Effects of different hormonal contraceptives in women's blood pressure values. Rev Bras Enferm. 2018;71(Supl.3):1453-43. http://dx.doi.org/10.1590/0034-71672017-0317. PMid:29972547.

22. Mello TS, Klen MS, Azevedo RB, Barradas FC, Nogueira LA, Ushijima NRM et al. Cardiovascular risk profile of a young adult women population assisted in primary care. Int J Cardiovasc Sci. 2021;34(4):372-82. http:// dx.doi.org/10.36660/ijcs.20200418.

23. WHO: World Health Organization. Global status report on alcohol and health 2018 [Internet]. Geneva: WHO; 2018 [citado 23 fev 2019]. Disponível em: https://www.who.int/substance_abuse/publications/ global_alcohol_report/en/

24. Lopes CS, Hellwig N, Silva GA, Menezes PR. Inequities in access to depression treatment: results of the Brazilian National Health Survey - PNS. Int J Equity Health. 2016;15(1):154. http://dx.doi.org/10.1186/ s12939-016-0446-1. PMid:27852278.

25. Jardim PCBV. Overweight, the cardiovascular risk of the century. Arq Bras Cardiol. 2019;113(2):185-7. http://dx.doi.org/10.5935/abc.20190171. PMid:31483014

26. NCD Risk Factor Collaboration (NCD-RisC). Trends in adult body-mass index in 200 countries from 1975 to 2014: a pooled analysis of 1698 population-based measurement studies with 19.2 million participants. Lancet. 2016;387(10026):1377-96. http://dx.doi.org/10.1016/S0140 6736(16)30054-X. PMid:27115820.

27. Costa Louzada ML, Martins AP, Canella DS, Baraldi LG, Levy RB, Claro RM et al. Ultra-processed foods and the nutritional dietary profile in Brazil. Rev Saude Publica. 2015;49:38. http://dx.doi.org/10.1590/ S0034-8910.2015049006132. PMid:26176747.

28. Malachias MVB, Souza WKSB, Plavnik FL, Rodrigues CIS, Brandão AA Neves MFT et al. 7 a Diretriz Brasileira de Hipertensão Arterial. Arq Bras Cardiol. 2016;107(3, Supl. 3):1-83. PMid:27819380.

29. Abegaz TM, Shehab A, Gebreyohannes EA, Bhagavathula AS, Elnour AA. Nonadherence to antihypertensive drugs: a systematic review and meta-analysis. Medicine. 2017;96(4):e5641. http://dx.doi.org/10.1097/ MD.0000000000005641. PMid:28121920.

30. Coyoy A, Guerra-Araiza C, Camacho-Arroyo I. Metabolism regulation by estrogens and their receptors in the central nervous system before and after menopause. Horm Metab Res. 2016;48(8):489-96. http://dx.doi. org/10.1055/s-0042-110320. PMid:27392117.

31. IDF:International Diabetes Federation. Diabetes atlas [Internet].8thed. Brussels: IDF; 2017. 144 p. [citado 23 fev 2019]. Disponível em: https://diabetesatlas. org/upload/resources/previous/files/8/IDF_DA_8e-EN-final.pdf

aArtigo extraído da dissertação de mestrado "Fatores de risco cardiovascular e práticas de cuidado entre mulheres: um estudo misto", de autoria de Gabriela
Oliveira, orientada por Maria Denise Schimith. Programa de Pós-Graduação em Enfermagem. Universidade Federal de Santa Maria. Ano de defesa 2019. 